\title{
Solution structures of intrinsically disordered dipeptide-repeats studied by SAXS and molecular structure simulation
}

\author{
Tien-Chang Lin, ${ }^{1 *}$ Kuei-Fen Liao ${ }^{2}$, Bing-Jun Lian ${ }^{1}$, Kai-Tai Lin ${ }^{1}$, Yu-Jen Chang ${ }^{3}$, Orion Shih ${ }^{2}$, Yi-Qi Yeh ${ }^{2}$, Yun-Ru \\ Chen $^{2,3}$ and U-Ser Jeng ${ }^{1,2^{\star}}$ \\ ${ }^{1}$ Department of Chemical Engineering, National Tsing Hua University, Hsinchu 30013, Taiwan; \\ ${ }^{2}$ National Synchrotron Radiation Research Center, Hsinchu 30076, Taiwan \\ ${ }^{3}$ Genomics Research Center, Academia Sinica, 115, Taiwan \\ bb91428tw@gmail.com; usjeng@nsrrc.org.tw
}

Often found in the gene chromosome 9 open reading frame 72 (C9ORF72) in the patients of familial frontotemporal dementia (a progressive disorder of the brain) and amyotrophic lateral sclerosis (muscles decreasing in size, resulting in difficulty in speaking, swallowing, and eventually breathing) are segments of abnormal dipeptide repeats, which serve as a signature of the diseases mentioned. Such dipeptide repeating of $10-1000$ times can be found in the brain or spinal cord of the patients, including toxic Glycine-Arginine $(\mathrm{GR})_{\mathrm{n}}$. Using the biological small-angle X-ray scattering beamline 13A at the Taiwan Photon Source (TPS), covering a wide range of scattering vector $q=0.01$ to $1.0 \AA^{-1}$, we observed gradually ordered solution structures of $(\mathrm{GR})_{\mathrm{n}}(\mathrm{n}=5,10$, $15,20,25,30$ ) when the $n$ value increases over 20 . The model structures of the dipeptide repeats reconstructed based on the SAXS data analysis combined with molecular simulation suggest a possible formation mechanism of the ordered structures. Effect of intervening Prolines into the GR dipeptide repeats is also observed. We note that up to date, there are no crystal structures available for the intrinsically disorder dipeptide repeats.

Keywords: Dipeptide repeats; Proline-Arginine; solution X-Ray Scattering; Molecular structural simulation 